\title{
Aminoacyl-tRNA synthetase deficiencies in search of common themes
}

\author{
Sabine A. Fuchs, MD, $\mathrm{PhD}^{1}$, Imre F. Schene, $\mathrm{MD}^{1}$, Gautam Kok, $\mathrm{BSc}^{1}$, Jurriaan M. Jansen, $\mathrm{MSc}^{1}$, \\ Peter G. J. Nikkels, MD, PhD², Koen L. I. van Gassen, PhD ${ }^{3}$, Suzanne W. J. Terheggen-Lagro, MD, PhD ${ }^{4}$, \\ Saskia N. van der Crabben, MD, PhD ${ }^{5}$, Sanne E. Hoeks, MD ${ }^{6}$, Laetitia E. M. Niers, MD, PhD ${ }^{7}$, \\ Nicole I. Wolf, MD, PhD ${ }^{8}$, Maaike C. de Vries, MD ${ }^{9}$, David A. Koolen, MD, PhD ${ }^{10}$, \\ Roderick H. J. Houwen, MD, PhD ${ }^{11}$, Margot F. Mulder, MD, PhD ${ }^{12}$ and Peter M. van Hasselt, MD, PhD ${ }^{1}$
}

Purpose: Pathogenic variations in genes encoding aminoacyltRNA synthetases (ARSs) are increasingly associated with human disease. Clinical features of autosomal recessive ARS deficiencies appear very diverse and without apparent logic. We searched for common clinical patterns to improve disease recognition, insight into pathophysiology, and clinical care.

Methods: Symptoms were analyzed in all patients with recessive ARS deficiencies reported in literature, supplemented with unreported patients evaluated in our hospital.

Results: In literature, we identified 107 patients with AARS, DARS, GARS, HARS, IARS, KARS, LARS, MARS, RARS, SARS, VARS, YARS, and QARS deficiencies. Common symptoms (defined as present in $\geq 4 / 13$ ARS deficiencies) included abnormalities of the central nervous system and/or senses (13/13), failure to thrive, gastrointestinal symptoms, dysmaturity, liver disease, and facial dysmorphisms. Deep phenotyping of 5 additional patients with unreported compound heterozygous pathogenic variations in IARS, LARS, KARS, and QARS extended the common phenotype with lung disease, hypoalbuminemia, anemia, and renal tubulopathy.

Conclusion: We propose a common clinical phenotype for recessive ARS deficiencies, resulting from insufficient aminoacylation activity to meet translational demand in specific organs or periods of life. Assuming residual ARS activity, adequate protein/amino acid supply seems essential instead of the traditional replacement of protein by glucose in patients with metabolic diseases.

Genetics in Medicine (2018) 21:319-330; https://doi.org/10.1038/ s41436-018-0048-y

Keywords: Aminoacyl-tRNA synthetase deficiency; Cytosolic translation; Clinical phenotype

\section{INTRODUCTION}

Protein translation is essential for all forms of life. Within this process, aminoacyl transfer RNA (tRNA) synthetases (ARSs) play an important role. This highly conserved ubiquitously expressed class of enzymes is responsible for correct coupling of amino acids to their cognate tRNAs, ${ }^{1-3}$ which base pair with messenger RNA (mRNA) triplets. ${ }^{4}$ This ensures incorporation of correct amino acids in the growing polypeptide chain during protein synthesis. Each proteinogenic amino acid is coupled to its corresponding tRNA by a specific ARS, ${ }^{1}$ as reflected in the ARS nomenclature (for example IARS for isoleucine-tRNA synthetase; LARS for leucine-tRNA synthetase). Because protein translation takes place in both the cytosol and mitochondria, mammalian cells possess cytosolic and mitochondrial ARSs. Mitochondrial ARSs are encoded by separate nuclear genes (nomenclature: ARS2), with the exception of KARS, GARS, and QARS, which encode both the cytosolic and mitochondrial ARSs. ${ }^{1}$

Over the past 15 years, an increasing proportion of ARS genes has been associated with human disease. In the first description, a heterozygous missense pathogenic variation in GARS was found to cause Charcot-Marie-Tooth disease type 2D, a peripheral axonal neuropathy. ${ }^{5}$ Subsequently, other dominant pathogenic variations in GARS, ${ }^{6-16}$ YARS,,${ }^{17,18}$ AARS, ${ }^{19-24} \mathrm{HARS}^{25}{ }^{25} \mathrm{KARS}^{26}$ and ${ }^{2}$ ARRS ${ }^{27}$ have been associated with peripheral axonal neuropathies. In the past 10 years, pathogenic variations in mitochondrial ARSs (DARS2, RARS2, EARS2, MARS2, FARS2, YARS2, SARS2,

${ }^{1}$ Department of Metabolic Diseases, Wilhelmina Children's Hospital, University Medical Center Utrecht, Lundlaan 6, Utrecht 3584 EA, The Netherlands; ${ }^{2}$ Department of Pathology, University Medical Center Utrecht, Lundlaan 6, Utrecht 3584 EA, The Netherlands; ${ }^{3}$ Department of Genetics, University Medical Centre Utrecht, Lundlaan 6, Utrecht 3584 EA, The Netherlands; ${ }^{4}$ Department of Pediatric Pulmonology, Academic Medical Center Amsterdam, Meibergdreef 9, Amsterdam 1105 AZ, The Netherlands; ${ }^{5}$ Department of Clinical Genetics, VU University Medical Center, De Boelelaan 1117, Amsterdam 1081HV, The Netherlands; ${ }^{6}$ Department of Neonatology, University Medical Center Utrecht, Lundlaan 6, Utrecht 3584 EA, The Netherlands; ${ }^{7}$ Department of Pediatrics, Maxima Medical Centre Veldhoven, De Run 4600, Veldhoven 5504 DB, The Netherlands; ${ }^{8}$ Department of Child Neurology, VU University Medical Center, De Boelelaan 1117, Amsterdam 1081HV, The Netherlands; ${ }^{9}$ Nijmegen Centre for Mitochondrial Disorders at Department of Pediatrics, Radboud University Nijmegen Centre, Nijmegen, The Netherlands; ${ }^{10}$ Department of Human Genetics, Radboud University Medical Center, Nijmegen 6500 HB, The Netherlands; ${ }^{11}$ Division of Pediatric Gastroenterology, Wilhelmina Children's Hospital, University Medical Center Utrecht, Lundlaan 6, Utrecht 3584 EA, The Netherlands; ${ }^{12}$ Department of Pediatrics, VU University Medical Center, De Boelelaan 1117, Amsterdam 1081HV, The Netherlands. Correspondence: Sabine A. Fuchs (S.Fuchs@umcutrecht.nl)

These authors contributed equally: Imre F. Schene, Gautam Kok. 


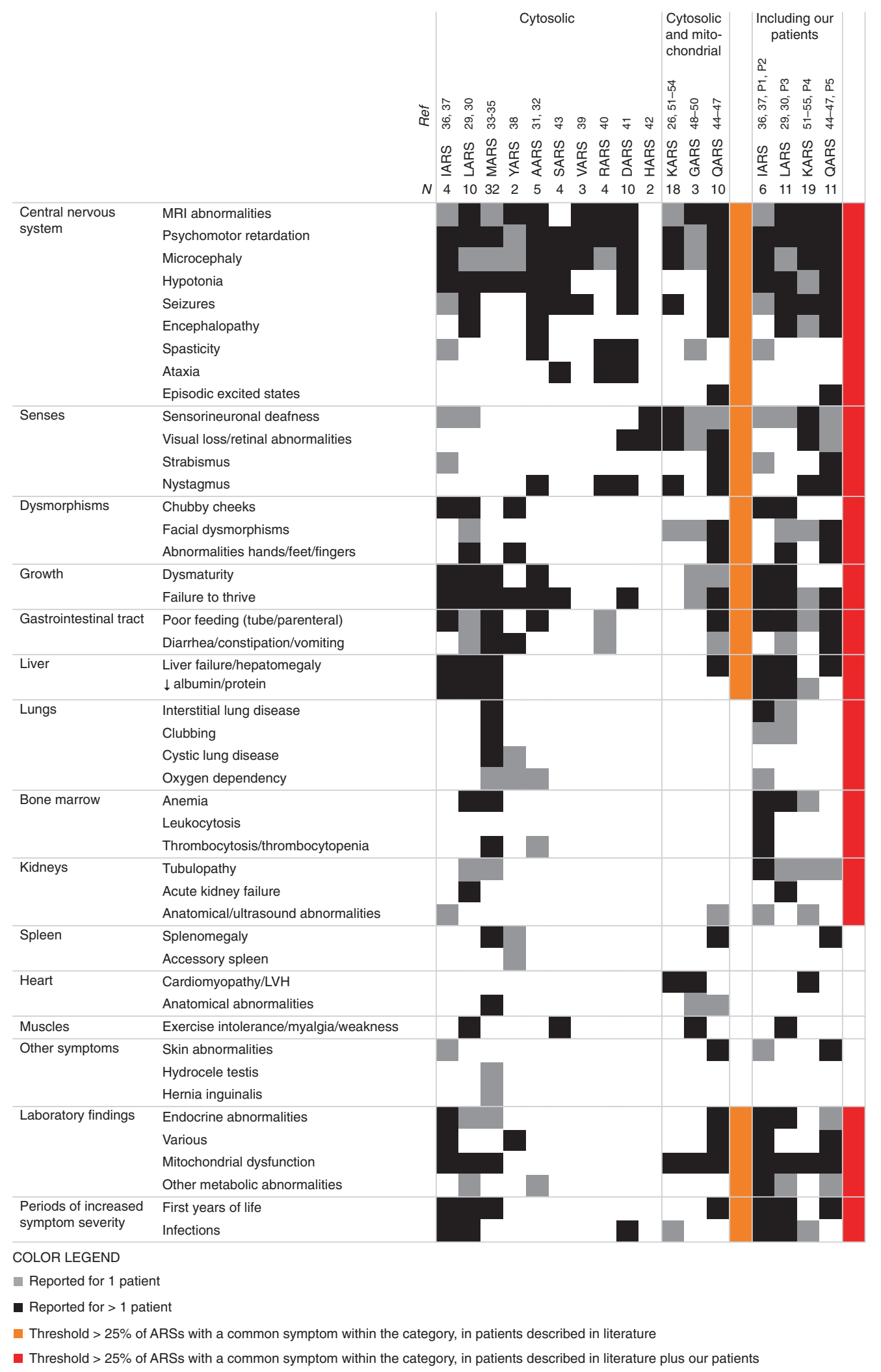

Fig. 1 Clinical symptoms of patients with autosomal recessive ARS deficiencies reported in literature (left part) and supplemented with P1-5 (right part). Gray squares represent symptoms reported for 1 patient, black squares symptoms reported for $>1$ patient. Categories of symptoms occurring in $>30 \%$ of the individual ARS deficiencies are marked in orange for patients reported in literature and in red after addition of P1-5. MRI magnetic resonance imaging, LVH left ventricular hypertrophy 
$A A R S 2$, and $H A R S 2)^{28}$ have emerged as a cause of human disease. The clinical presentation of these mitochondrial diseases was reported to depend on the affected ARS, with encephalopathy as the most common manifestation. Only in the past 5 years have homozygous and compound heterozygous pathogenic variations been identified in cytosolic LARS, ${ }^{29,30}$ AARS, ${ }^{31,32}$ MARS, ${ }^{33-35}{ }^{2} A R S,{ }^{36,37}$ YARS, ${ }^{38}$ VARS ${ }^{39}{ }^{29}$ ARS,${ }^{40}$ DARS ${ }^{41}{ }^{4}$ HARS, ${ }^{42}$ SARS, ${ }^{43}$ and in the combined cytosolic and mitochondrial QARS, ${ }^{44-47}$ $G A R S,{ }^{48-50}$ and $K A R S^{26,51-54}$ genes. The resulting autosomal recessive diseases show considerable clinical variability and involve numerous organs without apparent logic.

With the increasing number of diagnosed patients, insight in the pathophysiological mechanism is crucial for optimal clinical care. To this end, we reviewed literature for patients with autosomal recessive ARS deficiencies. We additionally deep phenotyped five patients with novel compound heterozygous pathogenic variations in IARS, $L A R S, K A R S$, and QARS and illustrate the common themes in disease presentation.

\section{MATERIALS AND METHODS}

A literature search was performed up to July 2017 in PubMed and Embase (search terms tRNA synthetase deficiency, ARS deficiency, (individual) ARS pathogenic variations) and through references in retrieved publications. Clinical details and laboratory results were retrieved from the manuscripts and supplemental material. The search was supplemented with all patients seen in our hospital with recessive ARS deficiencies diagnosed through exome sequencing. Exomes were enriched using Agilent SureSelect XT Human All Exon kit V5 and sequenced on a HiSeq sequencing system (Illumina). Reads were aligned to hg19 using Burrows-Wheeler Aligner. Variants were called using Genome Analysis Toolkit variant caller and annotated, filtered, and prioritized using the Bench NGS Lab platform (Agilent-Cartagenia, Leuven, Belgium) and/or an in-house designed "variant interface" and manual curation. Patients were evaluated according to our clinical practice and all laboratory analyses were performed in ISO 9001/ISO15189 accredited diagnostic laboratories. Clinical and laboratory findings were categorized. Findings reported in $>30 \%(\geq 4 / 13)$ of individual ARS deficiencies were considered common among ARS deficiencies.

\section{RESULTS}

We identified 107 children with homozygous or compound heterozygous pathogenic variations in 10 different cytosolic and 3 combined cytosolic and mitochondrial ARS genes (Fig. 1, Table S1). All reported ARS deficiencies were associated with affected central nervous system (CNS) and/ or senses (hearing/sight), most with failure to thrive $(10 / 13)$ and many with feeding problems (6/13), dysmaturity (5/13), and liver symptoms (4/13). Signs of mitochondrial dysfunction and facial dysmorphisms were common (6/13 and 4/13, respectively, including all three combined cytosolic and mitochondrial ARSs) and various endocrine abnormalities (4/13). Symptoms were most severe in the first year of life (4/13) and during infections and included acute liver failure and status epilepticus and/or encephalopathy (4/13).

We additionally identified five patients with pathogenic variations in $A R S$ genes (nomenclature according to Human Genome Variation Society [HGVS] guidelines):

$\mathrm{P} 1$, female, died at age 4 months, compound heterozygous pathogenic variations in IARS (NM_002161.5): c.1305G>C p.(Trp435Cys) and c.3377dup p.(Asn1126fs)

$\mathrm{P} 2$, male, now age 5 years, compound heterozygous pathogenic variations in IARS (NM_002161.5): c.1305G $>C$ p.(Trp435Cys) and c.3377dup p.(Asn1126fs). The parents originate from the same region as $\mathrm{P} 1$, which may suggest a founder effect for their common pathogenic variation P3, female, now age 5.5 years, compound heterozygous pathogenic variations in LARS (NM_020117.10): c.3420del p.(Ile1141fs) and c.1283C $>$ T p.(Pro428Leu)

P4, male, died at age 4 years, compound heterozygous pathogenic variations in KARS (NM_001130089.1): c1732_1744delG

GCATTGATCGAG, p.(Gly578SerfsX20) and c.683C> $\mathrm{T}$ p.(Pro228Leu)

P5, male, now age 4 years, compound heterozygous pathogenic variations in QARS (NM_005051.2): c.2084 +2_2084+3del p.(?) and c.793C > T p.(Arg265Cys)

Similar to the patients reported in literature, we observed CNS symptoms, growth/feeding problems, and liver disease (Fig. 1, Table S1).

\section{CNS symptoms}

$\mathrm{P} 1, \mathrm{P} 2$, and $\mathrm{P} 5$ were born and remained microcephalic (<-2SDS, Fig. 2). Head circumference at birth of P3-4 are unknown, but subsequent measurements were normal. CNS was most seriously affected in P4-5 with combined mitochondrial and cytosolic ARS deficiencies. Both patients had severe global developmental delays. P4 had an estimated developmental age of 4 months at age 4 years, involving eye contact, intentional laugh, recognition of parents, hypotonia, bipyramidal syndrome combined with extrapyramidal movements, but no rolling nor independent sitting. P5 had an estimated developmental age of 9 months at age 3.5 years, involving independent sitting and standing with support, but no crawling, language development, or interest in toys. Both patients had severe epilepsy. P4 had a permanent electroencephalogram (EEG) pattern of a (non) convulsive status epilepticus and from the age of 6 months jerky movements of the extremities, head, and/or eyes, somewhat improving with levetiracetam and clonazepam. P5 had a refractory epileptic encephalopathy despite ketogenic diet, deteriorating with vaccination/infections. In addition, $\mathrm{P} 4$ had severe perceptive hearing deficit, treated with cochlear implants and P5 severe occipital visual 

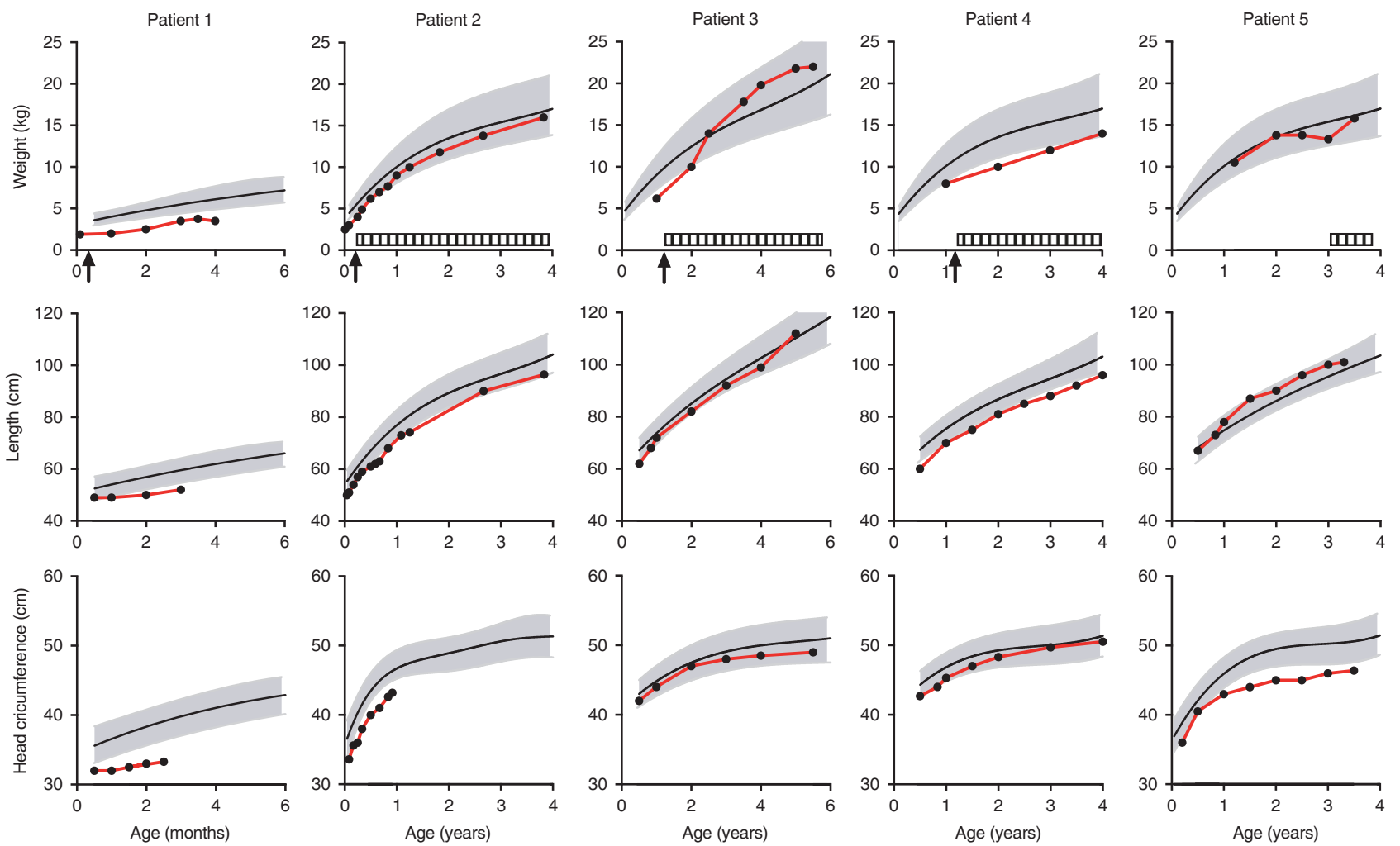

Fig. 2 Growth curves of weight, length, and head circumference of P1-5. Arrows indicate hospitalization due to feeding problems requiring nasogastric tube feeding (P1-4). Striped bars represent periods of feeding by percutaneous endoscopic gastrostomy (P2, P3, P5) or nasogastric tube (P4). Notice that the ranges of the $x$-axes are not synchronized between patients due to age differences

disturbance, with random eye movements and almost absent eye fixation or following.

Symptoms were milder in P1-3. Due to young age and hospitalization, development in P1 was difficult to judge. She was hypotonic, alert, made good eye contact, and passed her hearing test. P2-3 showed evidence of delayed global development (P2: global hypotonia, developmental quotients for global development, motoric skills, and verbal capacities ranging from 49 to 75 at age 3.5 years; P3: motor functioning p16, verbal IQ 81, performal IQ 70 at 5.5 years). P1-2 showed no evidence of epilepsy. P3 was hospitalized in her second and third year of life for three proven and one suspected status epilepticus, all triggered by infections. Electroencephalogram showed a focal left temporo-occipital lesion. Lamotrigine was started and no signs of epilepsy were noted after the age of 4 years.

Brain imaging of P3-5 showed distinct abnormalities (Fig. 3a). In P3, brain MRI showed periventricular white matter abnormalities (at age 13 months and 4.5 years), and focal cerebellar atrophy, without other neurodegenerative abnormalities or abnormalities of the thalami and basal ganglia. Magnetic resonance spectroscopy (MRS) showed elevated lactate peaks in white and gray matter. In P4, brain MRI at age 11 months showed hyperintense abnormalities in the substantia nigra. In P5, brain MRI was relatively normal at age 3 months, but displayed delayed white matter myelination, gradual loss of frontal periventricular white matter, and a relatively thin corpus callosum at age 1 year. At autopsy, microscopic evaluation of the brain of P1 (age 4 months) showed sparse white matter with decreased occipital myelination of $U$ fibers. Neuronal damage, presumably toxic, was seen in the basal ganglia and thalamus and minor recent hypoxic/ischemic damage in cerebellar neurons.

\section{Intrauterine growth restriction, feeding problems, and failure to thrive}

Intrauterine growth restriction was noted at 20 weeks of pregnancy in P1 and 3. P1-3 were born severely dysmature (P1: 1770 g; P2: 2250 g; P3: 1495 g, Fig. 2), P4 p2.3-5 (2800 g) and P5 P20-50 (3125 g). There were no signs of placental insufficiency or infections. In P1-4, the neonatal period was characterized by feeding problems and grossly insufficient growth, requiring hospitalization and nasogastric/duodenal tube feeding (P1: age $<1$ week, P2: 2 months, P3: 13 months, P4: 13 months), in P3 prompted by fasting intolerance $(4.5-10 \mathrm{~h})$. Percutaneous endoscopic gastrostomy was performed in P2, P3, and P5. Extensive analyses including gastrointestinal passage studies and esophagogastroduodenoscopy showed no anatomic, histologic abnormalities, nor malabsorption. With enteral feeding, growth normalized in P2, 3, and 5, but remained deficient in $\mathrm{P} 4$. 

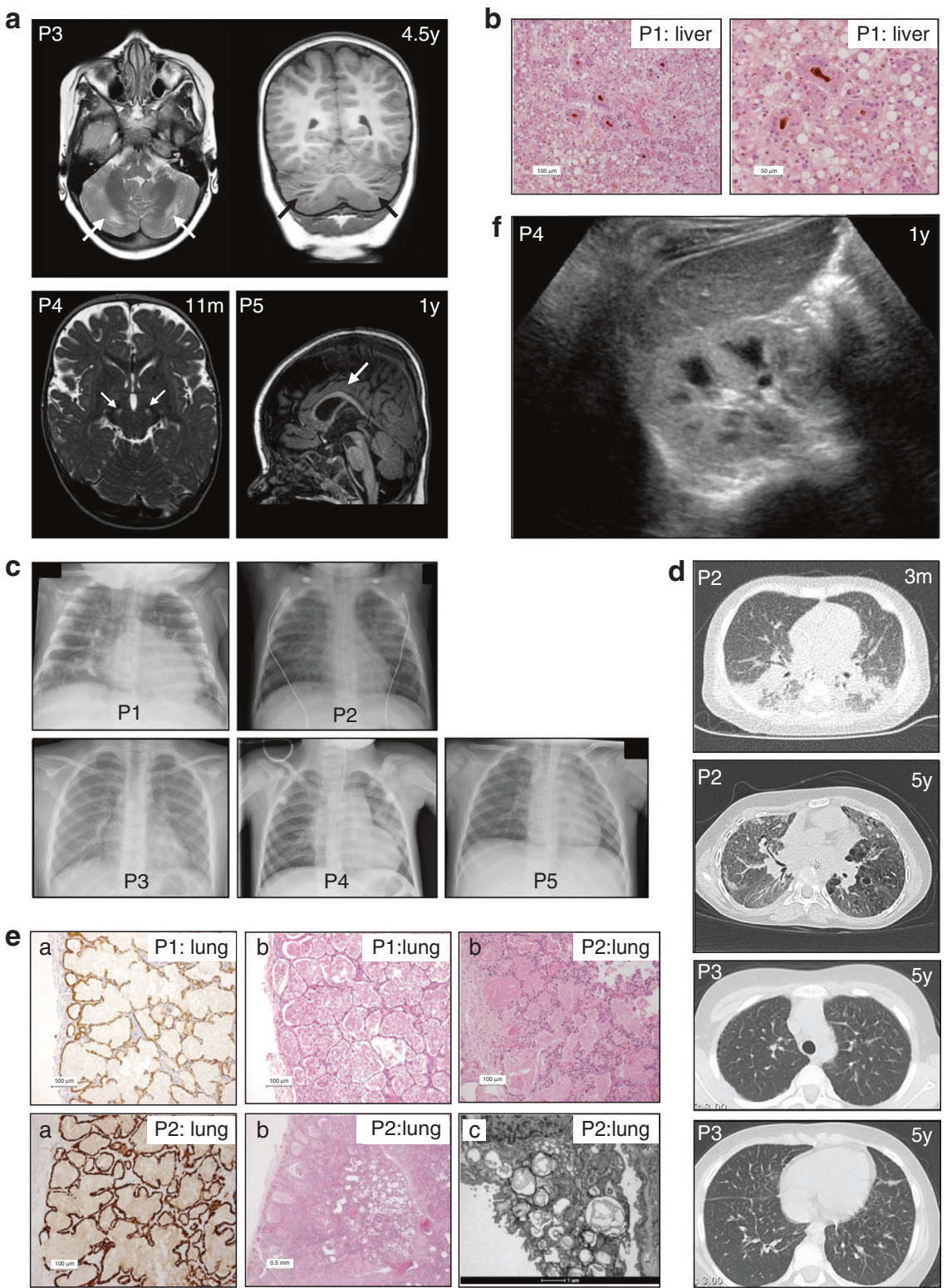

Fig. 3 Radiologic and histologic findings. (a) Brain magnetic resonance image (MRI) scans of P3, P4, and P5. T2-weighted axial and T1-weighted coronal MRI in P3 at age 4.5 years show focal atrophy of the cerebellar cortex. T2-weighted MRI in P4 at age 11 months shows abnormalities in the substantia nigra. T1-weighted MRI in P5 at age 1 year shows delayed myelination with a relatively thin corpus callosum. (b) Histology shows severe cholestasis and steatosis in liver tissue (P1) in H\&E staining (pseudorosette formation around bile plugs (brown). (c) Chest X-rays of P1-5. Interstitial abnormalities are visible in P1-P4. (d) Thoracic computed tomography (CT) scan in P2 at age 3 months shows extensive bilateral peribronchial consolidations, bronchus dilation, and subpleural ground glass consolidation with a remarkable dorso-basal distribution; at age 5 years it shows diffuse ground glass abnormalities, cystic lesions in a paraseptal-subpleural-bronchovascular distribution, and some thickening of interlobular septae. Thoracic CT scan in P3 at age 5 years shows thickening of interlobular septae in the upper and lower thorax. (e) Histology shows severe pulmonary alveolar proteinosis in lung tissue (P1 and P2) by staining for pankeratin marker CKAE1/3: (a: highlights the lining of the alveoli with reactive type 2 pneumocytes, $\mathbf{b}$ : H\&E staining; $\mathbf{b}$ : P1 and P2 alveoli are filled with a dense, eosinophilic, amorphous, protein-lipid precipitate; $\mathrm{P} 2$ shows granular material in multivesicular bodies and absent formation of lamellar bodies in type 2 pneumocytes, and c: electron microscopy; c: alveoli contain laminated annular structures [lamellar bodies]). (f) Ultrasound of the kidneys in P4 shows a hyperechogenic cortex of the kidneys. The global intensity of the kidney cortex versus medulla and liver is too intense

\section{Liver disease}

P1-3 had markedly elevated liver enzymes (Fig. 4) in the first year of life, normalizing with age in $\mathrm{P} 2$ and to a lesser extent in P3. There were no signs of known (metabolic) liver diseases. During infections, liver enzymes rose dramatically in these patients, accompanied by transient (P3) and refractory (P1) ascites in the context of progressive liver failure. Liver ultrasound imaging showed normal homogeneous aspect at age 1 month and progressive enlargement from age 2 months onwards in P1; a slightly enlarged, diffusely hyperechogenic liver and left lobe hypertrophy, with normalization of echogenicity from age 2.5 years onwards in P2; and an enlarged liver with multiple hypoechogenic masses at 13 months, confirmed with MRI abdomen and persistent at 

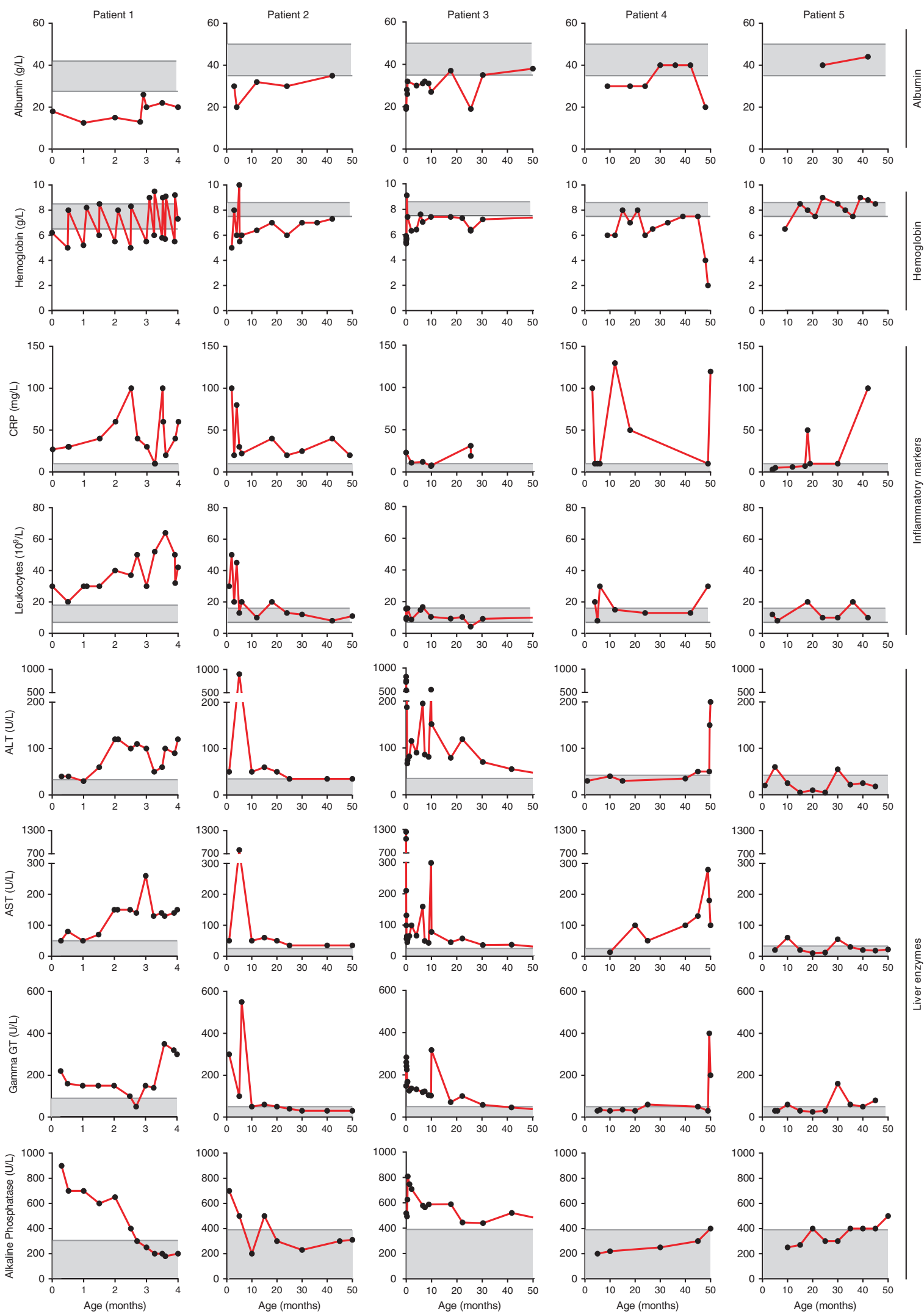

Fig. 4 Laboratory findings of P1-5. Notice that the ranges of the $x$-axes are not synchronized between patients due to age differences. ALT alanine transaminase, AST aspartate transaminase, CRP C-reactive protein, gamma-GT, gamma-glutamyltransferase 
age 5.5 years, with then also echogenic nodules in the pancreas in P3. Liver biopsy showed micro- and macrovesicular steatosis in $\mathrm{P} 1$ and combined steatosis and cirrhosis in P3. Liver steatosis, cholestasis, and extensive fibrosis were present at autopsy in P1 (Fig. 3b). Conversely, P4 only showed elevated liver enzymes in the final stage of his disease and P5 only during two episodes of antiepileptic drug introduction.

\section{Additional common clinical symptoms Hypoalbuminemia}

Blood albumin concentrations were severely decreased P1-4 in their first year of age and increased temporarily upon albumin infusions in P1-2 (Fig. 4). There were no signs of intestinal or renal protein loss. Protein electrophoresis showed proportional protein deficiency, suggestive of a general protein synthesis defect. In P2-4, hypoalbuminemia resolved spontaneously with age (P2: 11 months, P3: 18 months, and P4: 30 months, followed by severe hypoalbuminemia in his last episode of life). Albumin was only determined after age 17 months in P5 and was normal.

\section{Anemia}

Anemia was present in all five patients (Fig. 4) and hematocrit paralleled hemoglobin levels. Mean corpuscular volume was elevated in P1, P2, and P5 (up to 90-100, reference range: 73-87 fl), but low to normal in P3-4 (62-83 fl), while mean corpuscular hemoglobin was normal (1.75-2.25 fmol, P1 and P5), decreased to $1.3 \mathrm{fmol}$ (P2) or low (1.19-1.65 fmol; P3-4). Mean corpuscular hemoglobin concentration was normal in all patients. Reticulocytes were low $\left(13 \times 10^{9} / \mathrm{L}\right)$ in the first 3 months in $\mathrm{P} 1$ and rose to $180 \times 10^{9} / \mathrm{L}$ at 3.5 months, varied between 34 and $127 \times 10^{9} / \mathrm{L}$ in $\mathrm{P} 2$ and were normal in $\mathrm{P} 4-5$ $\left(25-120 \times 10^{9} / \mathrm{L}\right)$. The immature reticulocyte fraction was increased (to 0.5; normal range 0.09-0.31) and erythroblasts were present (to maximally $0.42 \times 10^{9} / \mathrm{L}$ ) in $\mathrm{P} 1, \mathrm{P} 2$, and $\mathrm{P} 4$. Morphology varied between normal and mild and inconsistent abnormalities. There were no signs of blood loss or hemolysis (no association with elevated bilirubin or lactate dehydrogenase (LDH) levels). Bone marrow aspiration in P2 showed a markedly elevated ratio of granulopoiesis to erythropoiesis (5:1). Taken together, these results imply aberrant erythropoiesis in the first year(s) of life in our patients. P1-2 were treated with repetitive erythrocyte transfusions; P3 with a transfusion followed by iron therapy until age 6 months. At age 11 months, she was still anemic while ferritin concentration was high (305 ug/L, reference range: $25-250 \mu \mathrm{g} / \mathrm{L}$ ); iron was started in P4 at age 14 months, compensating low ferritin concentrations $(5-10 \mu \mathrm{g} / \mathrm{l})$ and normalizing hemoglobin. With age, anemia gradually resolved.

\section{Inflammation}

In the first 6 months of life, P1 and P2 showed an inflammatory reaction with severe leukocytosis with predominantly neutrophils, varying thrombocytosis (P1: normal to $600 \times 10^{9} / \mathrm{L} ; \quad \mathrm{P} 2$ : $400-1400 \times 10^{9} / \mathrm{L} ;$ reference range: $\left.150-450 \times 10^{9} / \mathrm{L}\right)$, elevated C-reactive protein (CRP) and ferritin concentrations (P1: 680-1050 $\mu \mathrm{g} / \mathrm{L}$, reference range: $20-150 \mu \mathrm{g} / \mathrm{L}$ ) (Fig. 4). Treatment with corticosteroids temporarily normalized CRP and leukocytes in P1. After age 1 year, leukocytes and thrombocytes gradually normalized in P2. However, CRP remains elevated $(>15 \mathrm{mg} / \mathrm{L})$ up to his current age of 4.5 years. Bone marrow aspiration and blood smear showed myeloid and lymphoid dysplasia as seen in aberrant stimulation, without evidence of malignancies. P4 also displayed inflammatory reactions in the first month of life and during infections, with leukocytosis, thrombocytosis $\left(550-600 \times 10^{9} / \mathrm{L}\right)$, and elevated CRP, and complete normalization thereafter. P3 and P5 had normal CRP and leukocyte concentrations and adequate inflammatory response during infections.

\section{Pulmonary alveolar proteinosis}

Respiratory symptoms were most prominent in $\mathrm{P} 2$, with respiratory distress at age 2 months requiring high-flow nasal oxygen administration and two days of invasive ventilation. Thoracic chest X-ray and high-resolution CT (HR-CT) scan (Fig. 3c,d) showed extensive bilateral interstitial pulmonary abnormalities. Lung histology revealed pulmonary alveolar proteinosis (PAP) (Fig. 3e). He is still dependent on oxygen administration and recently developed cystic abnormalities (Fig. 3d). P1 required invasive ventilation during respiratory syncytial virus infection and could not be weaned from the ventilator nor oxygen. At autopsy, severe PAP was evident (Fig. 3e). P3 has been hospitalized once for pneumonia. She is easily fatigued, without overt respiratory symptoms. She has digital clubbing like her father. Aware of PAP in P1-2, chest X-rays were performed (Fig. 3c). Interstitial abnormalities, compatible with PAP, were noted and confirmed by thoracic HR-CT scan at age 5 years (Fig. 3d). P4 presented with fever, dyspnea, and respiratory insufficiency requiring ventilatory support at age 4 years. Chest X-ray showed signs of infection without clear interstitial pulmonary abnormalities (Fig. 3c). Further investigations were not conducted. P5 has no history of respiratory symptoms. Thoracic chest X-ray was performed once (Fig. 3c) upon presentation with fever and tachypnea. Symptoms resolved after antibiotic treatment.

\section{Metabolic abnormalities}

Analysis of urine from P1-2 showed increased excretion of galactose (maximal $1219 \mathrm{mmol} / \mathrm{mol}$ creatinine and 2534 $\mathrm{mmol} / \mathrm{mol}$ creatinine, respectively, reference range: $<838$ $\mathrm{mmol} / \mathrm{mol}$ creatinine) and galactitol (maximal $1082 \mathrm{mmol} /$ mol creatinine and $378 \mathrm{mmol} / \mathrm{mol}$ creatinine, respectively, reference range: $<107 \mathrm{mmol} / \mathrm{mol}$ creatinine), which normalized after lactose free feeding in P1 and spontaneously in P2. Enzyme activities involved in galactose breakdown and neonatal screening results for galactosemia were reviewed and normal in P1.

P1-2 and sporadically P4-5 displayed generalized aminoaciduria, which normalized with age in P2. Renal 


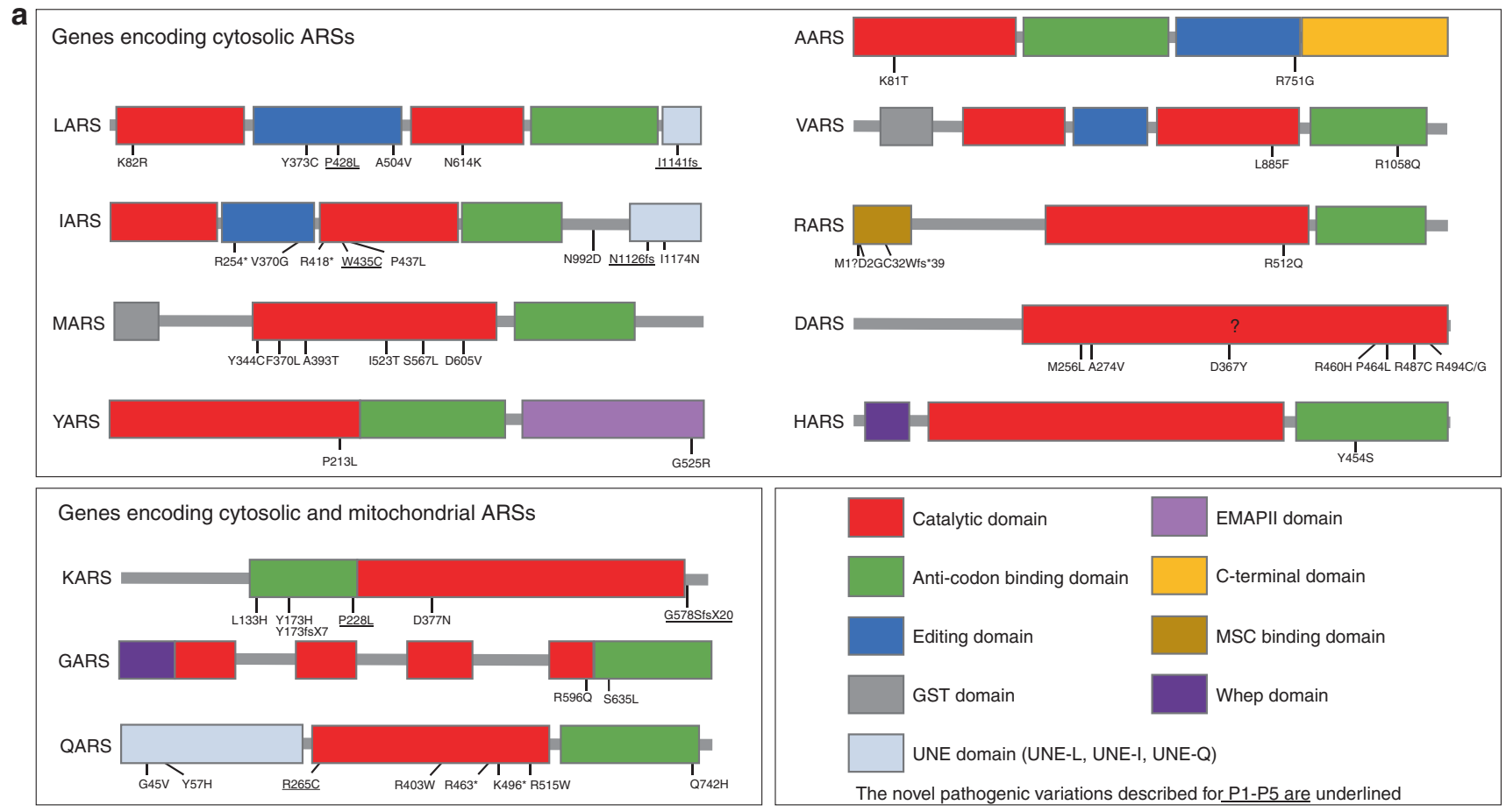

b

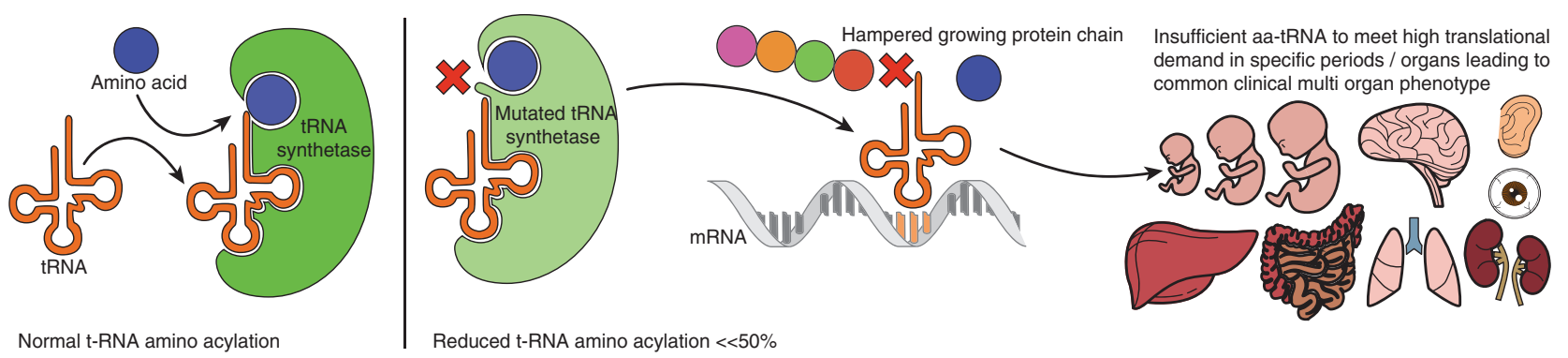

Fig. 5 Putative disease mechanism for autosomal recessive ARS deficiencies. (a) Overview of the pathogenic variations found in autosomal recessive ARS deficiencies, concentrated in the domains associated with the canonical function in protein translation. The novel pathogenic variations described for P1-P5 are underlined. (b) Schematic representation of the putative disease mechanism: insufficient aminoacylation to meet translational demand in specific organs or periods. tRNA transfer RNA

ultrasound in P4 showed bilateral increased renal cortex echogenicity (Fig. 3f), but no other signs of renal pathology.

Other metabolic abnormalities were only seen in individual patients, including for P1 increased cholesterol intermediates (cholestanol-7 and 8-dehydrocholesterol), putatively resulting from cholestasis or hypoalbuminemia and abnormal isoelectric focusing of serum transferrins, possibly secondary to liver failure or inflammation; for P2 increased urinary excretion of ethylmalonic acid (maximally $55 \mathrm{mmol} /$ mol creatinine at age 5 months, reference range: $<20 \mathrm{mmol} /$ mol creatinine) and bile acids, and elevated chitotriosidase blood concentration $(62.5 \mu \mathrm{mol} / \mathrm{hour} / \mathrm{L}$, reference range: $<37.1 \mu \mathrm{mol} / \mathrm{hour} / \mathrm{L}$ ) at age 3 months, putatively reflecting the inflammatory state; for P5 elevated blood and urine methylmalonic acid concentrations (maximally $3.11 \mu \mathrm{mol} / \mathrm{L}$; reference range: $0.12-0.25 \mu \mathrm{mol} / \mathrm{L}$; and $102 \mathrm{mmol} / \mathrm{mol} \mathrm{crea}-$ tinine; reference range: $0-20 \mathrm{mmol} / \mathrm{mol}$ creatinine, respectively) at age 3 months; there was no evidence of nutritional deficiencies with normal vitamin B12 and homocysteine concentrations. All abnormalities normalized with age.

Metabolic investigations were performed in $\mathrm{P} 3$ at age 13 months upon presentation with hypoglycemia, abnormal liver enzymes, and status epilepticus. She displayed elevated cerebrospinal fluid (CSF) lactate concentrations, but normal lactate/pyruvate ratio and alanine concentrations. Urinary lactate and pyruvate excretion were elevated, but organic acid excretion was normal. Oral glucose tolerance test showed a slight rise in lactate and low-normal ketone body production. Muscle biopsy revealed decreased substrate oxidation velocity and ATP $+\mathrm{CrP}$ production (10.9 nmol/h.mUCS, reference range: $15.4-30.2 \mathrm{nmol} / \mathrm{h} . \mathrm{mUCS})$, but normal enzyme activities of all respiratory chain enzymes, complex V, pyruvate dehydrogenase complex (PDHc) PDHc, and citrate synthetase. There were no signs of mitochondrial depletion syndromes or POLG, DGUOK, and MPV17 pathogenic variations. Glycogen storage diseases and lysosomal diseases 
(Niemann-Pick, lysosomal acid lipase deficiency [LALD]) were ruled out by enzyme assays and tyrosinemia and aberrant sugar or polyol metabolism by urinary analyses.

As might be expected, P4 showed evidence of mitochondrial dysfunction with elevated lactate levels in CSF (5.3 $\mathrm{mmol} / \mathrm{L}$, reference range: $1.1-2.1 \mathrm{mmol} / \mathrm{L}$ ) and plasma (3.5-10 $\mathrm{mmol} / \mathrm{L}$, reference range: $0-2.2 \mathrm{mmol} / \mathrm{L}$ ). Values rose after glucose challenge, with concomitantly increased urinary excretion of citric acid cycle intermediates. Lactate, pyruvate, alanine, and citric acid cycle intermediates dramatically increased in the final stage of disease. Muscle biopsy showed decreased substrate oxidation velocity and ATP $+\mathrm{CrP}$ production $(7.4 \mathrm{nmol} / \mathrm{h} . \mathrm{mUCS}$, reference values $15.4-30.2$ nmol/h.mUCS), but normal enzyme activities of the respiratory chain enzymes, complex $\mathrm{V}$, and citrate synthetase. Pathogenic variation analysis of POLG revealed no pathogenic variations. P5 showed normal lactate concentrations in blood and CSF, except during the period of epileptic encephalopathy (blood lactate concentration: 5.2 $\mathrm{mmol} / \mathrm{L})$.

\section{DISCUSSION}

Autosomal recessive ARS deficiencies represent a rapidly growing group of severe inherited diseases, involving multiple organs, currently without curative options. The rising number of recognized patients allows us to search for a potential common clinical phenotype, which might improve clinical recognition, insight into the disease mechanism, and clinical care. To this end, we compared clinical phenotypes of 112 patients with pathogenic variations in 10 cytosolic and 3 combined cytosolic and mitochondrial ARS genes, including all previously described patients with autosomal recessive ARS deficiencies we could identify in literature, supplemented with five new patients.

All ARS deficiencies were associated with CNS symptoms, including deficient hearing and/or sight, and most with failure to thrive, feeding, and gastrointestinal problems. Liver disease, chubby cheeks/facial dysmorphisms, various endocrine abnormalities, and mitochondrial dysfunction were reported for $>30 \%$ of the different ARS deficiencies (Fig. 1, orange) as was the pattern of severe symptoms in the first months of life and during infections. Identification of common clinical features from literature might underestimate true symptom overlap. Sometimes, not all clinical features were described, especially when studies were performed in specific cohorts of patients (HARS) ${ }^{42}$ or for specific clinical features (intellectual disability [SARS], ${ }^{43}$ leukoencephalopathy and leg spasticity [DARS] ${ }^{41}$ hypomyelination [RARS], ${ }^{40}$ neurogenetic disease [VARS] ${ }^{39}$ nonsyndromic hearing impairment $\left.[\mathrm{KARS}]\right) .{ }^{51} \mathrm{We}$ emphasize the importance of deep phenotyping in novel genetic diseases with five supplementary patients and encourage reporting all symptoms, also when not suspected as a core clinical feature. This allowed us to also classify lung disease, hypoalbuminemia, bone marrow, and kidney abnormalities as common (Fig. 1, red). Moreover, some symptoms might only be noted after specific diagnostic evaluation, as illustrated for restrictive lung disease in our IARS (P1) and LARS (P3) deficient patients.

Our findings suggest a strikingly common clinical phenotype for LARS, MARS, and IARS and to a slighter extent YARS and AARS deficiencies. Potentially, all symptoms reported for cytosolic ARS deficiencies represent the spectrum of a common clinical phenotype. Conversely, some features might be characteristic for specific ARS deficiencies. Nine cytosolic ARSs (IARS, LARS, MARS, QARS, KARS, RARS, DARS, and EPRS) form a multisynthetase complex (MSC). The major role ascribed to the MSC is to improve translational efficiency. Putatively, pathogenic variations in this subset of ARSs result in common symptoms based on deficient MSC function. Alternatively, symptoms might relate to specific amino acids (individually or to groups like branched chain amino acids), based on abundancy in specific proteins or to common (non)canonical amino acid or ARS functions. Combined cytosolic and mitochondrial ARS deficiencies obviously represent a specific subgroup, with mitochondrial dysfunction reflected in laboratory findings, muscle biopsy, and affected organs with high oxidative metabolism (brain, heart). Similarly, sensorineural hearing impairment is frequently seen in other mitochondrial ARS deficiencies, including LARS2, HARS2, RARS2, ${ }^{55}$ and IARS2. ${ }^{54}$ However, anemia and hypoalbuminemia in our KARS deficient patient and hepatosplenomegaly during infancy in QARS deficiencies ${ }^{46}$ might imply involvement of the cytosolic ARS deficiency. Conversely, although the multiorgan phenotype might suggest a mitochondriopathy, we did not find evidence for mitochondrial dysfunction caused directly by the cytosolic ARS deficiency.

The overlap in clinical phenotype between pathogenic variations in different $A R S$ s suggests a general disease mechanism. Pathogenic variations causing recessive ARS deficiencies generally reside in catalytic or anticodon binding domains of ARS genes (Fig. 5a). The most straightforward hypothesis therefore confers that aminoacylation is insufficient to meet translational demand in specific organs, especially during high demands in the first year of life and infections (Fig. 5b). Concurrently, aminoacylation activity was decreased in mutant alleles from patients with AARS, ${ }^{31,32}$ MARS,${ }^{33,34}$ IARS, $^{36}$ HARS $^{42}$ SARS, ${ }^{43}$ QARS, ${ }^{44,46}$ and KARS $^{26}$ deficiencies, but never to an enzyme activity of $<8 \%$ from normal (Table S2). The fact that heterozygous parents of patients are unaffected suggests that on the other hand there is some excess capacity in ARS activity.

Intrauterine growth restriction and failure to thrive seem closely related to a disease mechanism involving reduced aminoacylation activity, translational slowdown or inefficiency, and consequential decreased proliferation. Indeed, growth was diminished in complementation assays in yeast with mutant AARS, ${ }^{31}$ IARS,${ }^{36}$ MARS, ${ }^{34}$ GARS,${ }^{50}$ and KARS ${ }^{53}$ alleles. Similarly, hypoalbuminemia would well align with this disease mechanism, because albumin synthesis is very sensitive to amino acid depletion, particularly for leucine, isoleucine, and tryptophane. ${ }^{56}$ Moreover, organs most 
prominently affected in recessive ARS deficiencies are the organs with highest amino acid incorporation rates: liver $(2.4 \% / \mathrm{hr})$, lung $(1.0 \% / \mathrm{hr})$, brain $(0.6 \% / \mathrm{hr})$, and muscle $(0.4-0.7 \% / \mathrm{hr})^{57}$ or high proliferation rates (intestine). This also concurs with the prominence of symptoms during perinatal growth and infections, periods of high translational demand. It remains unclear why other highly proliferative organs like the skin are not affected, except in one patient with IARS deficiency and skin hyperelasticity ${ }^{37}$ and two sibs with QARS deficiency with a dry rough skin. ${ }^{46}$

PAP was previously thought to be a specific feature of MARS deficiency. ${ }^{33-35}$ We show that other recessive ARS deficiencies (IARS and LARS) can also cause PAP. Furthermore, our IARS deficient patient developed cystic abnormalities with time, as reported in YARS deficient patients. ${ }^{38}$ PAP results from the accumulation of lipoproteins in pulmonary alveoli, leading to restrictive lung disease and respiratory failure. ${ }^{58}$ Most commonly involved in the pathogenesis is defective granulocyte-macrophage colonystimulating factor (GM-CSF) receptor activation, either through GM-CSF autoantibodies ${ }^{59}$ or rare pathogenic variations in GM-CSF receptor genes. ${ }^{60}$ This leads to deficient alveolar macrophage maturation, which is essential for macrophages to clear and recycle surfactant. Interestingly, among patients with autoimmune antisynthetase syndromes, antibodies against ARSs strongly predict interstitial lung disease. ${ }^{61}$ In addition, PAP is also a clinical characteristic of lysinuric protein intolerance (LPI), a rare inherited metabolic disease $^{62}$ caused by a defective cationic amino acid transporter in the kidney, small bowel, lung, spleen, monocytes, and macrophages, resulting in leakage of cationic amino acids (arginine, ornithine, and lysine). Thus a shortage of amino acids, antibodies against ARSs, and genetic ARS deficiencies can all result in PAP, putatively through reduced aminoacylation and deficient translation to ensure adequate surfactant composition or homeostasis. Interestingly, LPI patients show considerable overlap in clinical phenotype with recessive ARS deficiencies, involving neurological impairment, failure to thrive, hepatosplenomegaly, PAP, renal tubulopathy, and hemophagocytic lymophohistiocytosis. ${ }^{62}$ The fact that both reduced amino acid concentrations and ARS deficiencies can result in common clinical symptoms concurs with insufficient aminoacylation for efficient translation as disease mechanism.

If reduced aminoacylation leads to symptoms in patients with recessive ARS deficiencies and if patients have some residual enzyme activity, then supplementation of the corresponding amino acid or high protein intake might improve symptoms. This is in sharp contrast with the traditional management of replacing protein by glucose in patients with metabolic hepatic disease, but aligns with the previous advice to patients with $L A R S$ pathogenic variations $^{29,30}$ to supply a minimum of $2.5 \mathrm{~g} / \mathrm{kg}$ of whole protein either enterally or parenterally while unwell. This is supported by the restoration of growth upon increased intake with tube feeding in P1-3 (Fig. 2). Similarly, the clinical phenotype in a patient with MARS deficiency improved after starting parenteral nutrition $^{33}$ and MARS aminoacylation activity and proliferation in yeast with mutant $M A R S$ alleles improved upon methionine supplementation. ${ }^{34}$

In conclusion, through deep phenotyping of five patients with novel compound heterozygous IARS, LARS, KARS, and $Q A R S$ pathogenic variations, we were able to add lung disease, hypoalbuminemia, anemia, and renal tubulopathy to the common phenotype we derived from literature for cytosolic ARS deficiencies, involving CNS abnormalities, growth restriction, liver symptoms, and facial dysmorphisms. This highlights the importance of deep phenotyping in patients with novel rare genetic diseases. Identification of a common clinical phenotype implies that recessive pathogenic variations in newly identified cytosolic ARSs may result in similar clinical symptoms, which might improve disease recognition and guide diagnostic work-up. Insufficient aminoacylation activity to meet high translational demand as common disease mechanism would also guide therapeutic care. As some residual enzyme activity seems essential for life, adequate supply of protein/amino acids is crucial, especially during periods of increased translational demand, including the first year of life and infections.

\section{ELECTRONIC SUPPLEMENTARY MATERIAL}

The online version of this article (https://doi.org/10.1038/s41436018-0048-y) contains supplementary material, which is available to authorized users.

\section{ACKNOWLEDGMENTS}

We acknowledge the patients, their parents, and caregivers for sharing the clinical details and funding contributions (P1). This work was further supported by Metakids funding (to SF), the Leading Ladies Fund from the Wilhelmina Children's Hospital (to SF), and a Clinical Fellows grant from The Netherlands Organisation for Health Research and Development Health Institute (4000703-97-13537 to SF).

\section{DISCLOSURE}

The authors declare no conflicts of interest.

\section{REFERENCES}

1. Antonellis A,Green ED. The role of aminoacyl-tRNA synthetases in genetic diseases. Annu Rev Genom Hum Genet. 2008;9:87-107.

2. Yao P, Fox PL. Aminoacyl-tRNA synthetases in medicine and disease EMBO Mol Med. 2013;5:332-43.

3. Storkebaum E. Peripheral neuropathy via mutant tRNA synthetases: Inhibition of protein translation provides a possible explanation. BioEssays. 2016;38:818-29. https://doi.org/10.1002/bies.201600052

4. Scheper GC, van der Knaap MS, Proud CG. Translation matters: protein synthesis defects in inherited disease. Nat Rev Genet. 2007;8:711-23.

5. Antonellis A, Ellsworth RE, Sambuughin N, et al. Glycyl tRNA synthetase mutations in Charcot-Marie-Tooth disease type 2D and distal spinal muscular atrophy type V. Am J Hum Genet. 2003;72:1293-9.

6. Sivakumar K, Kyriakides T, Puls I, et al. Phenotypic spectrum of disorders associated with glycyl-tRNA synthetase mutations. Brain. 2005;128:2304-14.

7. James PA, Cader MZ, Muntoni F, Childs AM, et al. Severe childhood SMA and axonal CMT due to anticodon binding domain mutations in the GARS gene. Neurology. 2006;67:1710-2. 
8. Del BoR, Locatelli F, Corti $S$, et al. Coexistence of CMT-2D and dista SMA-V phenotypes in an Italian family with a GARS gene mutation. Neurology. 2006;66:752-4.

9. Seburn $K L$, Nangle $L A$, Cox GA, et al. An active dominant mutation of glycyl-tRNA synthetase causes neuropathy in a Charcot-Marie-Tooth 2D mouse model. Neuron. 2006:51:715-26.

10. Rohkamm B, Reilly MM, Lochmüller $H$, et al. Further evidence for genetic heterogeneity of distal HMN type $\mathrm{V}, \mathrm{CMT} 2$ with predominant hand involvement and Silver syndrome. J Neurol Sci. 2007;263:100-6.

11. Abe A, Hayasaka K. The GARS gene is rarely mutated in Japanese patients with Charcot-Marie-Tooth neuropathy. I Hum Genet. 2009:54:310-2.

12. Lee HJ, Park J, Nakhro K, et al. Two novel mutations of GARS in Korean families with distal hereditary motor neuropathy type V. J Peripher Nerv Syst. 2012;17:418-21.

13. Eskuri JM, Stanley CM, Moore SA, et al. Infantile onset CMT2D/dSMA V in monozygotic twins due to a mutation in the anticodon-binding domain of GARS. J Peripher Nerv Syst. 2012;17:132-4.

14. Kawakami N, Komatsu K, Yamashita $\mathrm{H}$, et al. A novel mutation in glycyl-tRNA synthetase caused Charcot-Marie-Tooth disease type 2D with facial and respiratory muscle involvement. Rinsho Shinkeigaku. 2014;54:911-5

15. Sun $A$, Liu $X$, Zheng $M$, Sun $Q$, Huang $Y$, Fan $D$. A novel mutation of the glycyl-tRNA synthetase (GARS) gene associated with Charcot-Marie-Tooth type 2D in a Chinese family. Neurol Res. 2015;37:782-7.

16. Liao YC, Liu YT, Tsai PC, et al. Two novel de novo GARS mutations cause earlyonset axonal Charcot-Marie-Tooth disease. PLoS One. 2015;10:e0133423.

17. Jordanova A, Irobi J, Thomas FP, et al. Disrupted function and axonal distribution of mutant tyrosyl-tRNA synthetase in dominant intermediate Charcot-Marie-Tooth neuropathy. Nat Genet. 2006;38:197-202.

18. Hyun YS, Park HJ, Heo SH, et al. Rare variants in methionyl- and tyrosyltRNA synthetase genes in late-onset autosomal dominant Charcot-MarieTooth neuropathy. Clin Genet. 2014;86:592-4.

19. Latour P, Thauvin-Robinet C, Baudelet-Méry $C$, et al. A major determinant for binding and aminoacylation of tRNA ${ }^{\text {Ala }}$ in cytoplasmic alanyl-tRNA synthetase is mutated in dominant axonal Charcot-MarieTooth disease. Am J Hum Genet. 2010;86:77-82.

20. Lin K-P, Soong B-W, Yang C-C, et al. The mutational spectrum in a cohort of Charcot-Marie-Tooth disease type 2 among the Han Chinese in Taiwan. PLoS One. 2011;6:e29393.

21. Zhao Z, Hashiguchi $A$, Hu J, et al. Alanyl-tRNA synthetase mutation in a family with dominant distal hereditary motor neuropathy. Neurology. 2012:78:1644-9.

22. Bansagi B, Antoniadi T, Burton-Jones S, et al. Genotype/phenotype correlations in AARS-related neuropathy in a cohort of patients from the United Kingdom and Ireland. J Neurol. 2015;262:1899-908.

23. Motley WW, Griffin LB, Mademan I, et al. A novel AARS mutation in a family with dominant myeloneuropathy. Neurology. 2015:84:2040-7.

24. McLaughlin HM, Sakaguchi R, Giblin W, et al. A recurrent loss-of-function alanyl-tRNA synthetase (AARS) mutation in patients with charcot-marietooth disease type 2N (CMT2N). Hum Mutat. 2012;33:244-53.

25. Safka Brozkova D, Deconinck $T$, Beth Griffin $L$, et al. Loss of function mutations in HARS cause a spectrum of inherited peripheral neuropathies. Brain. 2015;138:2161-72.

26. McLaughlin HM, Sakaguchi R, Liu C, et al. Compound heterozygosity for loss-of-function lysyl-tRNA synthetase mutations in a patient with peripheral neuropathy. Am J Hum Genet. 2010;87:560-6.

27. Gonzalez $M$, McLaughlin $H$, Houlden $H$, et al. Exome sequencing identifies a significant variant in methionyl-tRNA synthetase (MARS) in a family with late-onset CMT2. I Neurol Neurosurg Psychiatry. 2013;84:1247-9.

28. Konovalova S, Tyynismaa H. Mitochondrial aminoacyl-tRNA synthetases in human disease. Mol Genet Metab. 2013;108:206-11.

29. Casey JP, McGettigan P, Lynam-Lennon N, et al. Identification of a mutation in LARS as a novel cause of infantile hepatopathy. Mol Genet Metab. 2012:106:351-8.

30. Casey JP, Slattery S, Cotter M, et al. Clinical and genetic characterisation of infantile liver failure syndrome type 1 , due to recessive mutations in LARS. J Inherit Metab Dis. 2015;38:1085-92.

31. Simons C, Griffin LB, Helman G, et al. Loss-of-function alanyl-tRNA synthetase mutations cause an autosomal-recessive early-onset epileptic encephalopathy with persistent myelination defect. Am J Hum Genet. 2015;96:675-81.
32. Nakayama T, Wu J, Galvin-Parton P, et al. Deficient activity of alanyl-tRNA synthetase underlies an autosomal recessive syndrome of progressive microcephaly, hypomyelination, and epileptic encephalopathy. Hum Mutat. 2017;38:1348-54.

33. van Meel $E$, Wegner DJ, Cliften $P$, et al. Rare recessive loss-of-function methionyl-tRNA synthetase mutations presenting as a multi-organ phenotype. BMC Med Genet. 2013;14:106.

34. Hadchouel A, Wieland T, Griese M, et al. Biallelic mutations of methionyltRNA synthetase cause a specific type of pulmonary alveolar proteinosis prevalent on Réunion Island. Am J Hum Genet. 2015;96:826-31.

35. Sun $Y, H u ~ G$, Luo J, et al. Mutations in methionyl-tRNA synthetase gene in a Chinese family with interstitial lung and liver disease, postnatal growth failure and anemia. J Hum Genet. 2017;62:647-51.

36. Kopajtich R, Murayama K, Janecke AR, et al. Biallelic IARS mutations cause growth retardation with prenatal onset, intellectual disability, muscular hypotonia, and infantile hepatopathy. Am J Hum Genet. 2016:99:414-22.

37. Orenstein N, Weiss K, Oprescu SN, et al. Bi-allelic IARS mutations in a child with intra-uterine growth retardation, neonatal cholestasis, and mild developmental delay. Clin Genet. 2017;91:913-7.

38. Nowaczyk MJM, Huang L, Tarnopolsky $M$, et al. A novel multisystem disease associated with recessive mutations in the tyrosyl-tRNA synthetase (YARS) gene. Am J Med Genet Part A. 2017:173:126-34.

39. Karaca E, Harel T, Pehlivan D, et al. Genes that affect brain structure and function identified by rare variant analyses of Mendelian neurologic disease. Neuron. 2015;88:499-513.

40. Wolf $\mathrm{NI}$, Salomons GS, Rodenburg RJ, et al. Mutations in RARS cause hypomyelination. Ann Neurol. 2014;76:134-9.

41. Taft RJ, Vanderver A, Leventer RJ, et al. Mutations in DARS cause hypomyelination with brain stem and spinal cord involvement and leg spasticity. Am J Hum Genet. 2013;92:774-80.

42. Puffenberger EG, Jinks RN, Sougnez $C$, et al. Genetic mapping and exome sequencing identify variants associated with five novel diseases. PLoS One. 2012;7:e28936.

43. Musante L, Püttmann L, Kahrizi K, et al. Mutations of the aminoacyltRNA-synthetases SARS and WARS2 are implicated in the etiology of autosomal recessive intellectual disability. Hum Mutat. 2017;38:621-36.

44. Zhang X, Ling J, Barcia G, et al. Mutations in QARS, encoding glutaminyltRNA synthetase, cause progressive microcephaly, cerebral-cerebellar atrophy, and intractable seizures. Am J Hum Genet. 2014:94:547-58.

45. Kodera $\mathrm{H}$, Osaka $\mathrm{H}$, lai $\mathrm{M}$, et al. Mutations in the glutaminyl-tRNA synthetase gene cause early-onset epileptic encephalopathy. J Hum Genet. 2015;60:97-101.

46. Leshinsky-Silver E, Ling J, Wu J, et al. Severe growth deficiency, microcephaly, intellectual disability, and characteristic facial features are due to a homozygous QARS mutation. Neurogenetics. 2017:18:141-6.

47. Datta A, Ferguson A, Simonson C, et al. Case report: QARS deficiency and favorable outcome following treatment of seizures with ketogenic diet. J Child Neurol. 2017;32:403-7.

48. McMillan HJ, Schwartzentruber J, Smith $A$, et al. Compound heterozygous mutations in glycyl-tRNA synthetase are a proposed cause of systemic mitochondrial disease. BMC Med Genet. 2014;15:36.

49. Nafisinia M, Riley LG, Gold WA, et al. Compound heterozygous mutations in glycyl-tRNA synthetase (GARS) cause mitochondrial respiratory chain dysfunction. PLoS One. 2017:12:e0178125.

50. Oprescu SN, Chepa-Lotrea X, Takase R, et al. Compound heterozygosity for loss-of-function GARS variants results in a multisystem developmental syndrome that includes severe growth retardation. Hum Mutat. 2017:38:1412-20

51. Santos-Cortez RLP, Lee K, Azeem Z, et al. Mutations in KARS, encoding lysyl-tRNA synthetase, cause autosomal-recessive nonsyndromic hearing impairment DFNB89. Am J Hum Genet. 2013;93:132-40.

52. McMillan HJ, Humphreys $P$, Smith $A$, et al. Congenital visual impairment and progressive microcephaly due to lysyl-transfer ribonucleic acid (RNA) synthetase (KARS) mutations. J Child Neurol. 2015;30:1037-43.

53. Verrigni $D$, Diodato $D$, Di Nottia $M$, et al. Novel mutations in KARS cause hypertrophic cardiomyopathy and combined mitochondrial respiratory chain defect. Clin Genet. 2017:91:918-23.

54. Kohda M, Tokuzawa $Y$, Kishita $Y$, et al. A comprehensive genomic analysis reveals the genetic landscape of mitochondrial respiratory chain complex deficiencies. PLoS Genet. 2016;12:e1005679. 
55. Glamuzina E, Brown $R$, Hogarth $K$, et al. Further delineation of pontocerebellar hypoplasia type 6 due to mutations in the gene encoding mitochondrial arginyl-tRNA synthetase, RARS2. J Inherit Metab Dis. 2012;35:459-67.

56. Hutson SM, Stinson-Fisher C, Shiman R, Jefferson LS. Regulation of albumin synthesis by hormones and amino acids in primary cultures of rat hepatocytes. Am J Physiol. 1987;252(3 pt 1): E291-8.

57. Shahbazian FM, Jacobs M, Lajtha A. Rates of protein synthesis in brain and other organs. Int J Dev Neurosci. 1987;5:39-42.

58. de Blic J. Pulmonary alveolar proteinosis in children. Paediatr Respir Rev. 2004;5:316-22.
59. Uchida K, Beck DC, Yamamoto T, et al. GM-CSF autoantibodies and neutrophil dysfunction in pulmonary alveolar proteinosis. $N$ Engl J Med. 2007;356:567-79.

60. Dirksen U, Nishinakamura R, Groneck $P$, et al. Human pulmonary alveolar proteinosis associated with a defect in GM-CSF/L-3/IL-5 receptor common beta chain expression. J Clin Invest. 1997;100:2211-7.

61. Marie I, Josse S, Decaux O, et al. Comparison of long-term outcome between anti-Jo1- and anti-PL7/PL12 positive patients with antisynthetase syndrome. Autoimmun Rev. 2012;11:739-45.

62. Mauhin W, Habarou F, Gobin S, et al. Update on lysinuric protein intolerance, a multi-faceted disease retrospective cohort analysis from birth to adulthood. Orphanet J Rare Dis. 2017;12:3. 